\title{
Blocking TBK1 alleviated radiation-induced pulmonary fibrosis and epithelial-mesenchymal transition through Akt-Erk inactivation
}

\author{
Hongjin Qu', Lei Liu', Zhe Liu', Hongran Qin', Zebin Liao', Penglin Xia', Yanyong Yang ${ }^{1}$, Bailong Li',
} Fu Gao ${ }^{1}$ and Jianming Cai ${ }^{1}$

\begin{abstract}
As a common serious complication of thoracic radiotherapy, radiation-induced pulmonary fibrosis (RIPF) severely limits radiation therapy approaches. Epithelial-mesenchymal transition (EMT) is a direct contributor to the fibroblast pool during fibrogenesis, and prevention of EMT is considered an effective strategy to inhibit tissue fibrosis. Our previous study revealed that TANK-binding kinase 1 (TBK1) regulates EMT in lung cancer cells. In the present study, we aimed to investigate the therapeutic potential of targeting TBK1 to prevent RIPF and EMT progression. We found radiationinduced EMT and pulmonary fibrosis in normal alveolar epithelial cells and lung tissues. TBK1 knockdown or inhibition significantly reversed EMT in vivo and in vitro and attenuated pulmonary fibrosis and collagen deposition. Moreover, we observed that TBK1 was elevated in a time- and dose-dependent manner by radiation. Meanwhile, radiation also induced time- and dose-dependent activation of AKT and ERK, each of whose inhibitors suppressed radiation-induced EMT. Intriguingly, silencing of TBK1 with shRNA also blocked the radiation-induced activation of AKT and ERK signaling. The ERK inhibitor did not obviously affect the expression of TBK1 or phosphorylated AKT, while AKT inhibition suppressed activation of ERK without changing the expression of TBK1. Finally, we found that a TBK1 inhibitor inhibited inflammatory cytokine expression in a RIPF model and Amlexanox protected normal cells and mice from ionizing radiation. In conclusion, our results indicate that the TBK1-AKT-ERK signaling pathway regulates radiation-induced EMT in normal alveolar epithelial cells, suggesting that TBK1 is a potential target for pulmonary fibrosis prevention during cancer radiotherapy.
\end{abstract}

\section{Introduction}

Lung cancer is the most common malignant cancer with high incidence and (especially non-small cell lung cancer (NSCLC)) has become the leading cause of cancer-related death $^{1,2}$. Radiotherapy, an effective treatment modality for thorax-associated neoplasms, is recommended as a mainstay in the treatment of NSCLC ${ }^{3,4}$. However, the risk of radiation-induced lung injury (RILI) in normal tissues hampers the efficacy of lung cancer radiotherapy.

\footnotetext{
Correspondence: Fu Gao (gaofusmmu@163.com) or Jianming Cai (cjm882003@163.com) or Bailong Li (libailong2013@163.com) 'Department of Radiation Medicine, Faculty of Naval Medicine, Second Military Medical University, 800, Xiangyin Road, 200433 Shanghai, P. R. China

These authors contributed equally: Hongjin Qu, Lei Liu, Zhe Liu
}

Accordingly, radiation-induced pulmonary fibrosis (RIPF), the adverse late effect of RILI, limits further application of radiotherapy with increasing radiation doses ${ }^{3-6}$. However, there is no available treatment strategies against RILI, and the underlying mechanism remains unclear ${ }^{7,8}$.

Epithelial-mesenchymal transition (EMT) plays a critical role in pathological fibrosis in many tissues. It was also reported that EMT occurs in idiopathic and experimental lung fibrosis ${ }^{9,10}$. Activated fibroblasts originating from alveolar epithelial cells through EMT produce collagen and extracellular matrix proteins in the lung interstitium, which results in lung fibrosis ${ }^{11-14}$. Radiation-induced EMT may play an important role in RIPF. Furthermore, overexpression

\section{(c) The Author(s) 2019}

(cc) Open Access This article is licensed under a Creative Commons Attribution 4.0 International License, which permits use, sharing, adaptation, distribution and reproduction cc in any medium or format, as long as you give appropriate credit to the original author(s) and the source, provide a link to the Creative Commons license, and indicate if changes were made. The images or other third party material in this article are included in the article's Creative Commons license, unless indicated otherwise in a credit line to the material. If material is not included in the article's Creative Commons license and your intended use is not permitted by statutory regulation or exceeds the permitted use, you will need to obtain permission directly from the copyright holder. To view a copy of this license, visit http://creativecommons.org/licenses/by/4.0/. 
of EMT-associated genes or promotion of radiation-induced EMT accelerates the incidence of RIPF ${ }^{15,16}$.

EMT is characterized by downregulation of epithelial proteins, such as E-cadherin, and acquisition of mesenchymal markers, including vimentin and $\alpha$-smooth muscle actin $(\alpha \text {-SMA })^{17}$. The regulation of classical EMT centers on the activities of major transcription factors, such as SNAI1, SNAI2, ZEB1, ZEB2, and TWIST1, which have been described and reviewed extensively ${ }^{18,19}$. TANK-binding kinase 1 (TBK1) has been identified as a downstream effector of miR-200c, which inhibits EMT by directly targeting ZEB1 and ZEB2, and our previous findings revealed that TBK1 signaling regulates radiation-induced EMT by controlling GSK3 $\beta$ phosphorylation and ZEB1 expression in lung cancer cells ${ }^{14,20,21}$. However, the exact role of TBK1 in radiation-induced EMT, especially in normal alveolar epithelial cells and tissues, still needs further exploration.

In recent studies, the AKT and ERK signaling pathways have been demonstrated to be the cardinal signaling programs mediating EMT in cancer cells ${ }^{22-25}$. It is well known that TBK1 can activate AKT through direct phosphorylation $^{26,27}$. Furthermore, it was revealed that radiation-induced EMT in alveolar type II epithelial cells is mediated by the ERK/GSK3 $\beta /$ Snail pathway ${ }^{13}$. We hypothesized that TBK1 might regulate radiation-induced EMT through the AKT signaling pathway or ERK signaling pathway. In this study, we explored the possible mechanisms associated with TBK1 in radiation-induced EMT in normal alveolar epithelial cells. Our data indicate that TBK1 may act as an upstream trigger of AKT and ERK via phosphorylation to affect the radiation-induced EMT process in normal alveolar epithelial cells, suggesting that TBK1 is a potential target for treatment of RIPF.

\section{Materials and methods}

\section{Cell culture and treatment}

Cells of the rat alveolar type II epithelial cell line RLE6TN were purchased from the American Type Culture Collection (Manassas, VA, USA) and routinely maintained in Dulbecco's modified Eagle's medium (DMEM)/ F12 (Gibco, Grand Island, NY, USA) with 10\% fetal bovine serum (Gibco), $100 \mathrm{IU} / \mathrm{ml}$ penicillin, and $100 \mu \mathrm{g} / \mathrm{ml}$ streptomycin (Invitrogen, CA, USA) at $37^{\circ} \mathrm{C}$ with $5 \%$ $\mathrm{CO}_{2}$. For some conditions, cells were pretreated with inhibitors for $2 \mathrm{~h}$ before irradiation.

\section{Mice and treatments}

Female C57BL/6 mice, 7 weeks of age, were purchased from the Experimental Animal Center of Chinese Academy of Sciences (Shanghai, China). Mice were randomly divided into four groups as follows: group 1, nonirradiated control (Control); group 2, irradiation + dimethyl sulfoxide (DMSO) (infrared (IR)); group 3, irradiation + amlexanox (IR + Amlexanox); group 4, irradiation + prednisone (IR + Prednisone). All mice were housed under standard laboratory conditions and were allowed to acclimate to the animal center for 1 week prior to treatment. Amlexanox was obtained from Abcam (Cambridge, MA, USA) and given orally 3 days before irradiation at a dose of $50 \mathrm{mg} / \mathrm{kg} /$ day and maintained until 4 weeks after irradiation. Prednisone (SHANGHAIZZBIOCO, Ltd., Shanghai, China) was administered orally at $5 \mathrm{mg} / \mathrm{kg} / \mathrm{day}$ postirradiation for 4 weeks.

\section{Irradiation}

Cells and mice were irradiated with $\gamma$-rays using a ${ }^{60} \mathrm{Co}$ irradiator (Faculty of Naval Medicine, Second Military Medical University, China). Cells were irradiated with a single dose of $0,2,4,6,8$, or $10 \mathrm{~Gy}$ at a dose rate of $1.8 \mathrm{~Gy} /$ min. After anesthetization with $10 \%$ choral hydrate $(350 \mathrm{mg} / \mathrm{kg})$, the mice were put in a holder designed to immobilize anesthetized mice so that only the whole thorax was exposed to the beam. Mice received a single dose of $20 \mathrm{~Gy}$ (thoracic irradiation) at a dose rate of $1 \mathrm{~Gy} / \mathrm{min}$. All irradiations were performed at room temperature.

\section{Western blot analysis}

We used Proteo-JETTM Mammalian Cell Lysis Reagent (Thermo) to lyse cells. Crude lysates were centrifuged at $14,000 \mathrm{~g}$ for $20 \mathrm{~min}$ at $4{ }^{\circ} \mathrm{C}$. Lysate proteins were mixed with loading buffer and denatured by heating at $100^{\circ} \mathrm{C}$ for $10 \mathrm{~min}$. Proteins were resolved by SDS-PAGE and transferred to a PVDF membrane (Millipore, Billerica, MA, USA), blocked with $5 \%$ nonfat milk, and then incubated with primary antibodies overnight at $4{ }^{\circ} \mathrm{C}$ on a shaking table. Primary antibodies targeting E-cadherin (1:1000), Vimentin (1:1000), AKT (1:1000), AKT-pS473 (1:1000), AKT-pT308 (1:1000), ERK (1:1000), p-ERK (1:1000) (Cell Signaling Technology, Danvers, MA, USA), $\alpha$-SMA (1:1000), TBK1 (1:1000) (Abcam, Cambridge, MA, USA), and GAPDH (Beyotime, China) were used. Then, the blots were incubated with the secondary antibody, HRP-conjugated IgG (Beyotime, dilution 1:5000), for $1 \mathrm{~h}$ at room temperature. Specific bands for each protein were detected with an Image Quant LAS4000 imager (GE Healthcare Life Science, Pittsburgh, PA) using Amersham ECL (Millipore). Densitometric analysis of expression was performed using ImageJ software (National Institutes of Health, Bethesda, MD). The expression of each molecule was normalized to GAPDH expression.

\section{Hematoxylin-eosin and Masson's trichrome staining}

The right lung of the mice was harvested and fixed in $4 \%$ neutral paraformaldehyde and then embedded in paraffin. Tissue specimens were sectioned at a thickness of $5 \mathrm{~mm}$ and conventionally stained with hematoxylineosin (H\&E) and Masson's trichrome stain for histological 
examination. Fibrosis grade was evaluated blindly using a semiquantitative scoring system.

\section{Hydroxyproline assay}

The concentration of hydroxyproline was measured with a hydroxyproline assay kit (Nanjing Jiancheng Bioengineering Institute, Nanjing, China) according to the manufacturer's protocol. Hydroxyproline content was calculated according to total lung weight and is expressed as micrograms in the lung.

\section{Clonogenic assay}

The radiation protective effect of amlexanox was assessed with clonogenic assays. The cells were trypsinized, counted, and seeded in $60-\mathrm{mm}$ culture dishes in two sets of three for each dose of radiation. Amlexanox, at a final concentration of $25 \mu \mathrm{M}$, was added to the cell dishes $1 \mathrm{~h}$ before irradiation and was discarded 4 days after irradiation. Sufficient numbers were seeded to ensure that approximately 30-100 macroscopic colonies would appear in each plate after 10-14 days. Colonies were stained with $0.5 \%$ gentian violet in methanol and counted. The plating efficiency (PE) for each dose was calculated by dividing the number of colonies by the number of cells plated and expressing the result as a percentage. The surviving fraction was calculated by dividing the PE of the treatment by the PE of the appropriate unirradiated control.

\section{Cell proliferation assay}

Cell proliferation was determined using Cell Counting Kit-8 (Dojindo, Kumamoto, Japan). Cells were suspended and seeded into 96 -well plates at $5 \times 10^{3}$ cells/well. Then, $48 \mathrm{~h}$ after irradiation, cell proliferation was assessed with a CCK-8 assay.

\section{Apoptosis analysis}

Apoptosis of cells treated with or without amlexanox and irradiation was determined by Annexin V-FITC (AV) and PI staining. The cells were plated in six-well plates at a density of $10^{5}$ cells per well and allowed to attach for 24 h. The cells were pretreated with and without $25 \mu \mathrm{M}$ amlexanox before irradiation. After $24 \mathrm{~h}$, the cells were harvested by trypsin digestion, washed with precooled phosphate-buffered saline (PBS) twice, and resuspended. The cells were stained with AV and PI at room temperature for $15 \mathrm{~min}$ in a dark room according to the Annexin V-FITC Apoptosis Detection Kit (BD Pharmingen, San Diego, CA) instructions.

\section{Immunofluorescence staining}

Cells cultured in six-well plates were irradiated with a single dose of $8 \mathrm{~Gy}$ of ${ }^{60} \mathrm{Co} \gamma$-rays. After $1 \mathrm{~h}$ or $48 \mathrm{~h}$, cells were washed with PBS and fixed in $4 \%$ paraformaldehyde for $20 \mathrm{~min}$. Then, we washed cells with
PBS and permeabilized the plasma membrane of cells with $0.1 \%$ Triton $\mathrm{X}-100$ for $30 \mathrm{~min}$ at room temperature. Cells were blocked with $5 \%$ bovine serum albumin (BSA) for $1 \mathrm{~h}$ and incubated with E-cadherin, Vimentin (Cell Signaling Technology), $\alpha$-SMA, TBK1, or p-AKT (Abcam) antibodies at $4{ }^{\circ} \mathrm{C}$ overnight. After being washed with PBS, the sections were incubated with Alexa Fluor 488-conjugated anti-mouse (Invitrogen) and Texas red-conjugated anti-rabbit (Vector Laboratories, Burlingame, CA, USA) antibodies at room temperature for $30 \mathrm{~min}$. Nuclei were counterstained with 4',6-diamidino-2-phenylindole (DAPI), and the slides were analyzed using a fluorescence microscope (Nikon, Tokyo, Japan). For the lung tissue, after deparaffinization and antigen retrieval, antibodies against E-cadherin and $\alpha$-SMA were mixed and used for immunofluorescence staining.

\section{Enzyme-linked immunosorbent assay}

Bronchi alveolar lavage fluid (BALF) was collected, and interleukin-4 (IL-4) and interferon- $\gamma$ (IFN- $\gamma$ ) levels were determined using a commercially available ELISA (enzyme-linked immunosorbent assay) kit according to the manufacturer's instructions. The OD value was determined at $450 \mathrm{~nm}$ using a Varioskan LUX Multimode Microplate Reader (Thermo Fisher Scientific Inc. Waltham, MA, USA) and calculated at the linear portion of the curve. BALF cytokine levels were measured using a commercial ELISA kit from Anogen (Mississauga, Ontario, Canada).

\section{Mouse survival analysis}

To determine the radioprotective property of amlexanox, mice were divided into the following two experimental groups: group 1, radiated (whole body) only animals $(n=10,20)$, animals were treated with vehicle (DMSO, $0.2 \mathrm{~mL}$, p.o.) $1 \mathrm{~h}$ before irradiation; group 2, 50 $\mathrm{mg} / \mathrm{kg}$ amlexanox + irradiated animals $(n=10,20)$, animals were treated with $50 \mathrm{mg} / \mathrm{kg}$ amlexanox $(0.2 \mathrm{~mL}$, p.o. $)$ $1 \mathrm{~h}$ before irradiation. After exposure, the mice were maintained at the animal facility, and mortality and morbidity was observed for 30 days.

\section{Lentiviral short hairpin RNA (shRNA) production/infection}

Rat TBK1 shRNA (CTGGGTGAGATTTCAGACATA) and scrambled shRNA (TTCTCCGAACGTGTCACGT) lentiviruses (made from the vector GV248) were generated by BioLink (Shanghai, China). TBK1 shRNA lentiviral vectors were used for knockdown of TBK1. Cells were seeded at $1 \times 10^{5}$ cells/well into six-well plates and infected with lentiviral particles using polybrene $(10 \mathrm{mg} / \mathrm{mL})$. After infection, virus-containing medium was replaced with normal medium, and then cells were selected with puromycin $(2 \mathrm{mg} / \mathrm{mL})$. 


\section{Statistical analysis}

Statistical analyses were performed using Prism 5.0 software (GraphPad). All quantitative data are presented as the mean \pm SEM and were obtained from at least three independent experiments. For normalization, sham control values were set to $100 \%$, and values for irradiated animals are displayed as a percent of the sham control values. Student's two-tailed unpaired $t$-tests were used to compare differences between two groups. One-way analysis of variance (ANOVA) followed by Newman-Keuls multiple comparison tests were used to compare more than two groups. Two-way ANOVA with post hoc Bonferroni multiple comparison tests were used to compare groups split on two independent variables. Statistical significance was set at $P<0.05$.

\section{Results}

TBK1 upregulation participates in radiation-induced EMT

Rat type II alveolar RLE-6TN cells were irradiated with a single dose of $0,2,4,6,8$, or 10 Gy $\gamma$-rays, and cell morphology was observed at $0,24,48$, and $72 \mathrm{~h}$ postirradiation. We found that $70 \%$ of the cells changed from a cuboidal appearance to a swollen and elongated morphology with extended pseudopodia after irradiation, especially at $48 \mathrm{~h}$ postirradiation with $8 \mathrm{~Gy} \gamma$-rays (Fig. 1a). To test if these changes were in line with EMT-associated proteins, we used western blotting to analyze expression of the epithelial marker E-cadherin and mesenchymal markers Vimentin and $\alpha$-SMA. A decrease in the protein level of E-cadherin and an increase in vimentin and $\alpha$ SMA were observed in cells after irradiation (Fig. 1b, c). Western blotting data revealed significant variation in the markers at 48 and $72 \mathrm{~h}$ postirradiation compared with the nonirradiated group (Fig. 1b). Intriguingly, changes in EMT-associated proteins in cells irradiated with $10 \mathrm{~Gy}$ were less marked than in those irradiated with $8 \mathrm{~Gy}$ (Fig. 1c). Moreover, the changes in EMT-associated markers were also confirmed by immunofluorescence staining (Fig. 1d). Taken together, these data suggest that irradiation likely induces EMT in RLE-6TN cells, especially at $48 \mathrm{~h}$ postirradiation and a $\gamma$-ray dose of $8 \mathrm{~Gy}$.

To investigate the role of TBK1 in EMT in normal type II alveolar epithelial cells, we first determined the radiation responsive property of TBK1. We subjected RLE-6TN cells to a single dose of 8 Gy and found a timedependent increase in the TBK1 protein level, especially after $1 \mathrm{~h}$ (Fig. 2a). Then, we observed a significant dosedependent increase in TBK1 protein from 2 to $10 \mathrm{~Gy}$ (Fig. 2b). To further validate the upregulation of TBK1, we conducted an immunofluorescence assay, which showed more fluorescence after 8 Gy irradiation at $48 \mathrm{~h}$ in contrast with the nonirradiated control (Fig. 2c). After that, we generated TBK1-specific shRNA to silence the expression of TBK1, and a significant reduction was observed in TBK1 expression (Fig. 2d). We found that most TBK1 knockdown cells exhibited a changed morphology, from a swollen, elongated morphology with extended pseudopodia to a cuboidal appearance at $48 \mathrm{~h}$ postirradiation (Fig. 2e). Moreover, TBK1 knockdown increased the expression of the epithelial marker E-cadherin and decreased the expression of the mesenchymal markers vimentin and $\alpha$-SMA after irradiation (Fig. 2f). Overall, overexpression of TBK1 contributed to radiation-induced EMT of RLE-6TN cells, and the loss of TBK1 expression may reverse this phenotype, suggesting that TBK1 may play an important role in radiation-induced EMT in normal alveolar epithelial cells.

\section{TBK1-dependent AKT phosphorylation is critical for radiation-induced EMT}

It is known that the AKT signaling pathway plays an important role in mediating EMT and is directly activated by TBK1 in cancer cells. Therefore, we aimed to test whether radiation could activate AKT and to determine its role in radiation-induced EMT. As shown in Fig. 3a, b, we found that AKT was significantly phosphorylated on both S473 and T308 at 1-3 h postirradiation and observed a dose-dependent increase in AKT activation with 2-8 Gy $\gamma$-ray treatment at $1 \mathrm{~h}$ postirradiation. Phosphorylation of AKT was further confirmed by immunofluorescence analysis (Fig. 3c). Then, to examine whether AKT participates in radiation-induced EMT in RLE-6TN cells, we preincubate cells with the selective AKT inhibitor PF04691502 for $2 \mathrm{~h}$ before irradiation. As shown in Fig. 3d, PF-04691502 remarkably inhibited phosphorylation of $\mathrm{AKT}$ at $1 \mathrm{~h}$ postirradiation with $8 \mathrm{~Gy}$ irradiation. The cell morphology again showed a cuboidal appearance at $48 \mathrm{~h}$ postirradiation when treated with the AKT inhibitor (Fig. 3e). The protein levels of E-cadherin, vimentin, and $\alpha$-SMA were effectively modulated by PF-04691502 at $48 \mathrm{~h}$ postirradiation (Fig. 3f), which indicated that blocking of AKT inhibited radiation-induced EMT in RLE-6TN cells.

Then, we investigated the possibility of a relationship between TBK1 and AKT. As shown in Fig. 4a, TBK1 shRNA blocked AKT phosphorylation on both S473 and T308, but the level of total AKT was not altered. Furthermore, silencing of TBK1 decreased the activation of AKT induced by 8 Gy irradiation at $1 \mathrm{~h}$ postirradiation (Fig. 4b). In contrast, the AKT inhibitor PF-04691502 had little effect on the protein level of TBK1, even after irradiation (Fig. 4c). These data indicate that AKT might be downstream of TBK1, suggesting that TBK1 may promote radiation-induced EMT through the AKT signaling pathway. 


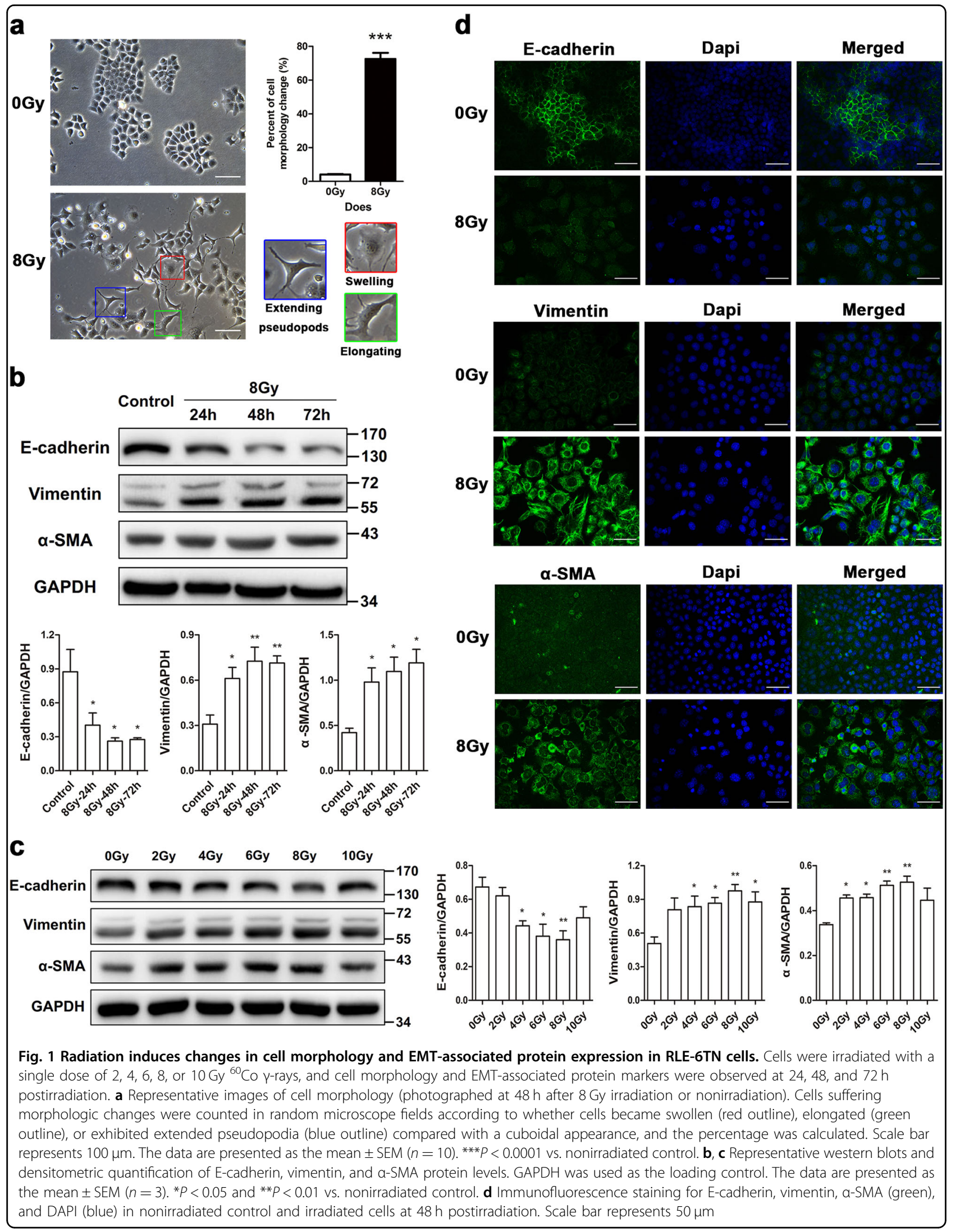




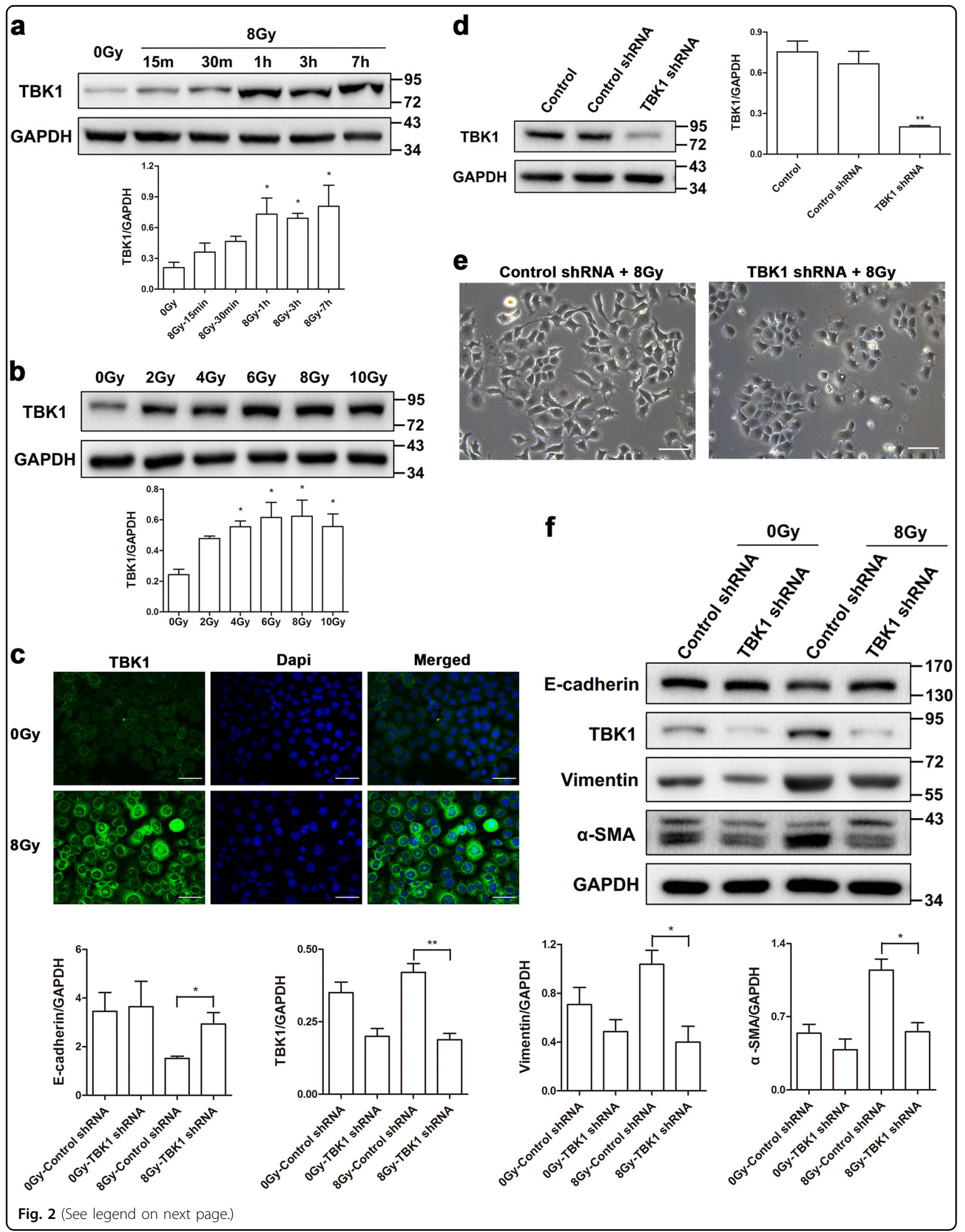


(see figure on previous page)

Fig. 2 Radiation induced TBK1 expression and knockdown of TBK1 attenuates radiation-induced EMT. a, b Representative western blots and densitometric quantification of TBK1 protein levels at different times and with different radiation doses. $\mathbf{c}$ Immunofluorescence staining for TBK1 (green) and DAPI (blue) in nonirradiated control and irradiated cells at $1 \mathrm{~h}$ postirradiation. Scale bar represents $50 \mu \mathrm{m}$. $\mathbf{d}$ Representative western blot and densitometric quantification of TBK1 following infection with TBK1-specific shRNA lentivirus to confirm target suppression. e Phase contrast microscopy images of cells expressing TBK1-specific shRNA and control shRNA at $48 \mathrm{~h}$ after $8 \mathrm{~Gy}$ irradiation. Scale bar represents $100 \mu \mathrm{m}$. $\mathbf{f}$ Western blot analysis of E-cadherin, vimentin, and a-SMA expression in TBK1 knockdown RLE-6TN cells. GAPDH was used as the loading control in all the above western blot analyses. All data are presented as the mean \pm SEM $(n=3)$. ${ }^{*} P<0.05$ and ${ }^{* *} P<0.01$ vs. nonirradiated control

TBK1 is required for radiation-induced ERK activation and EMT

It was also revealed that ERK signaling was involved in EMT. Therefore, we infer that there may be some connection between TBK1 and ERK in mediating radiationinduced EMT. We observed that ERK was activated by irradiation in a time-dependent and dose-dependent manner. As revealed in Fig. 5a, ERK was significantly phosphorylated at 3 and $7 \mathrm{~h}$ after treatment with $8 \mathrm{~Gy}$ irradiation. Consistently, ERK phosphorylation was also observed in a dose-dependent manner ranging from 2 to $10 \mathrm{~Gy}$, particularly with 6 and $8 \mathrm{~Gy}$ irradiation (Fig. 5b). The ERK selective inhibitor SCH772984 markedly blocked radiation-induced phosphorylation of ERK (Fig. 5c), and thus reversed the EMT phenotype in irradiated cells (Fig. 5d).

To investigate whether radiation-induced phosphorylation of ERK requires TBK1, we examined the phosphorylation of ERK in TBK1 knockdown cells. TBK1 knockdown restrained the basal level of phosphorylated ERK (Fig. 5e) and sharply inhibited radiation-induced phosphorylation of ERK at $3 \mathrm{~h}$ postirradiation (Fig. 5f). However, blocking ERK did not affect the protein level of TBK1 (Fig. 5g). Thus, ERK might be downstream of TBK1. These data indicate that the radiation-induced EMT in RLE-6TN cells is mediated, at least in part, via the TBK1-ERK signaling pathway.

\section{AKT-mediated ERK signaling downstream of TBK1 is critical for radiation-induced EMT}

We found that AKT and ERK signaling downstream of TBK1 may be necessary for radiation-induced EMT in RLE-6TN cells. Further, we investigated the relationship between AKT and ERK signaling in this biological process. Western blot analyses showed that phosphorylation of ERK was significantly decreased in irradiated RLE-6TN cells pretreated with the AKT inhibitor PF-04691502, whereas the levels of total ERK remained unchanged (Fig. 6a, b). This result indicated that AKT inhibition could override the radiation-induced activation of ERK. Then, we used the ERK inhibitor SCH772984 to confirm whether blocking ERK could attenuate radiation-induced AKT phosphorylation. However, there was no difference in the phosphorylated AKT protein level between nonirradiated control cells and those pretreated with the inhibitor (Fig. 6c, d). Nevertheless, SCH772984 did not obviously affect radiation-induced phosphorylation of AKT (Fig. 6c, d). Although there was some complex crosstalk between AKT and ERK signaling, these data suggest that AKT might act upstream of ERK in radiationinduced EMT in RLE-6TN cells.

\section{The TBK1 inhibitor Amlexanox alleviated radiation- induced pulmonary injury and fibrosis}

EMT is a critical process in radiation-induced lung fibrosis, which is one of the most important complications of lung cancer radiotherapy. Because TBK1 was found to play a critical role in EMT, we investigated the potential therapeutic role of TBK1 in RILI. We used the TBK1 inhibitor Amlexanox and found that Amlexanox obviously attenuated lung injury (Fig. 7a) and collagen deposition (Fig. 7c) at 1 week, 2 weeks, 4 weeks, 3 months, 6 months, and 12 months after irradiation. Quantitative data showed that Amlexanox significantly reduced lung fibrosis (Fig. 7b) and collagen deposition in lung tissues (Fig. 7d). Then, we measured the hydroxyproline content in lung tissues from different groups and found that Amlexanox significantly reduced the level of hydroxyproline after IR (Fig. 7e). In terms of lung fibrosis, the protective effects of Amlexanox were better than that in the prednisone group, the therapeutic medicine presently used for radiation-induced pulmonary injury (RIPI).

\section{Amlexanox inhibited EMT in lung tissues after irradiation}

To examine the role of TBK1 in radiation-induced EMT in vivo, we assessed the expression of EMT-related markers in different groups after irradiation. Our data showed that local lung irradiation resulted in obvious EMT changes, such as downregulation of E-cadherin and upregulation of Vimentin and $\alpha$-SMA. We found that Amlexanox significantly inhibited the decrease in Ecadherin and the upregulation of $\alpha$-SMA at 3, 6 , and $12 \mathrm{~m}$ after irradiation (Fig. 8a-f). The expression levels of these proteins were confirmed by western blot analysis (Fig. 8g-i). Inflammatory cytokines are important factors in radiation-induced lung fibrosis, and we checked the level of IL-4 and IFN- $\gamma$ in BALF. Our data showed that Amlexanox significantly inhibited the radiation-induced increase in the levels of these cytokines (Fig. 8j, k). 

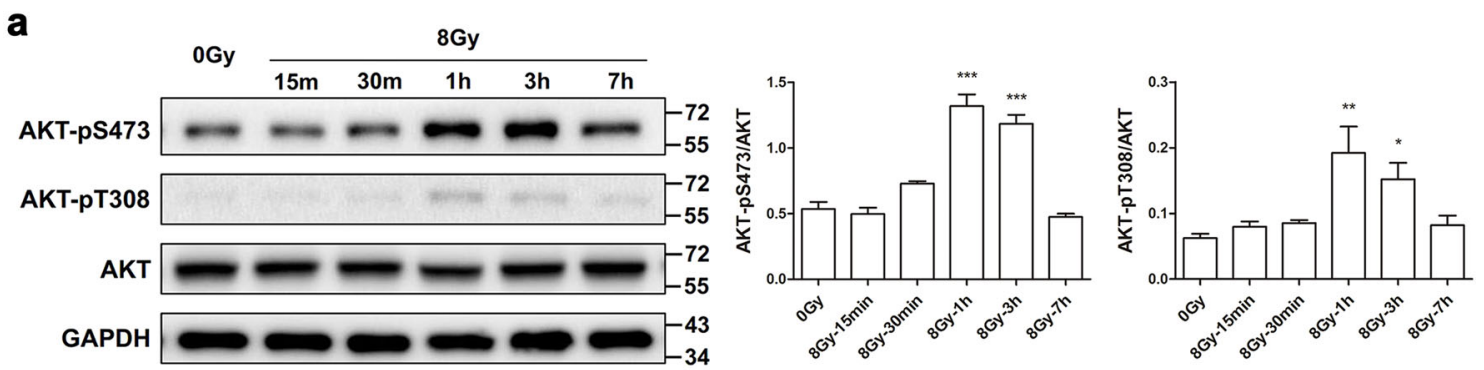

b
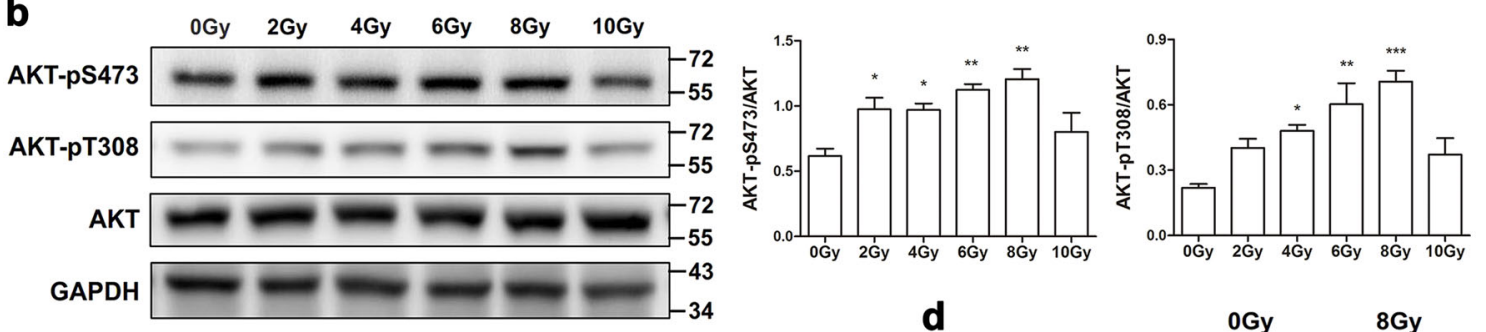

C
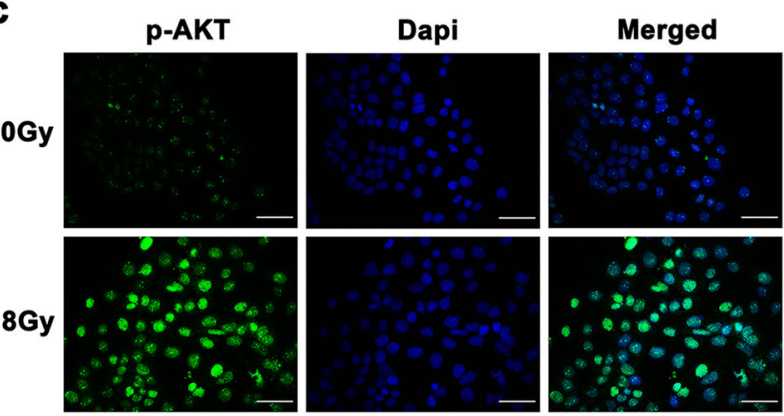

e

Control + 8Gy

PF-04691502 + 8Gy
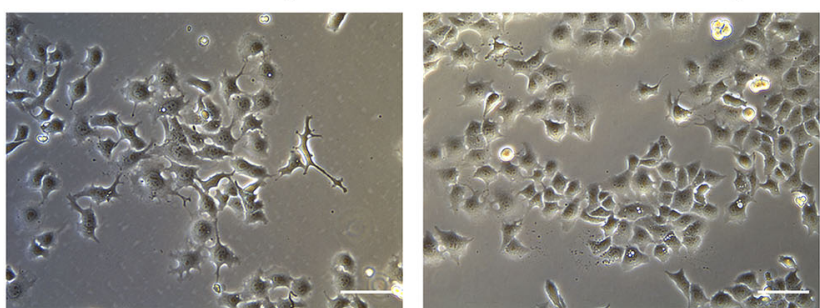

d
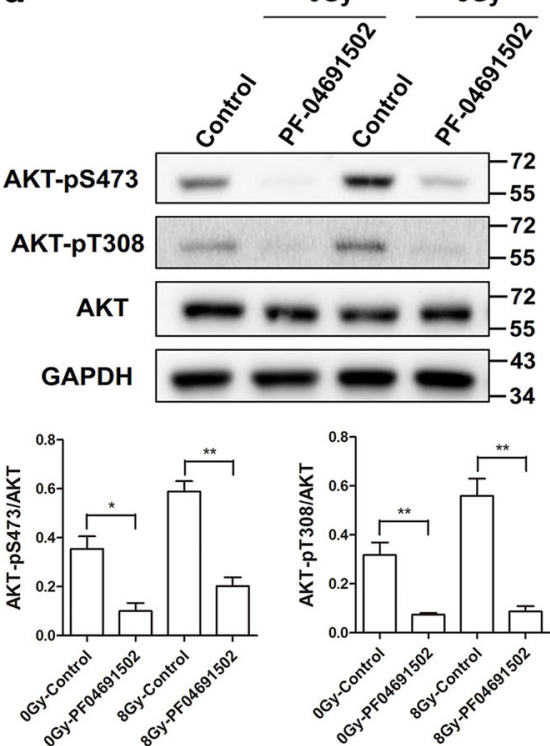

f
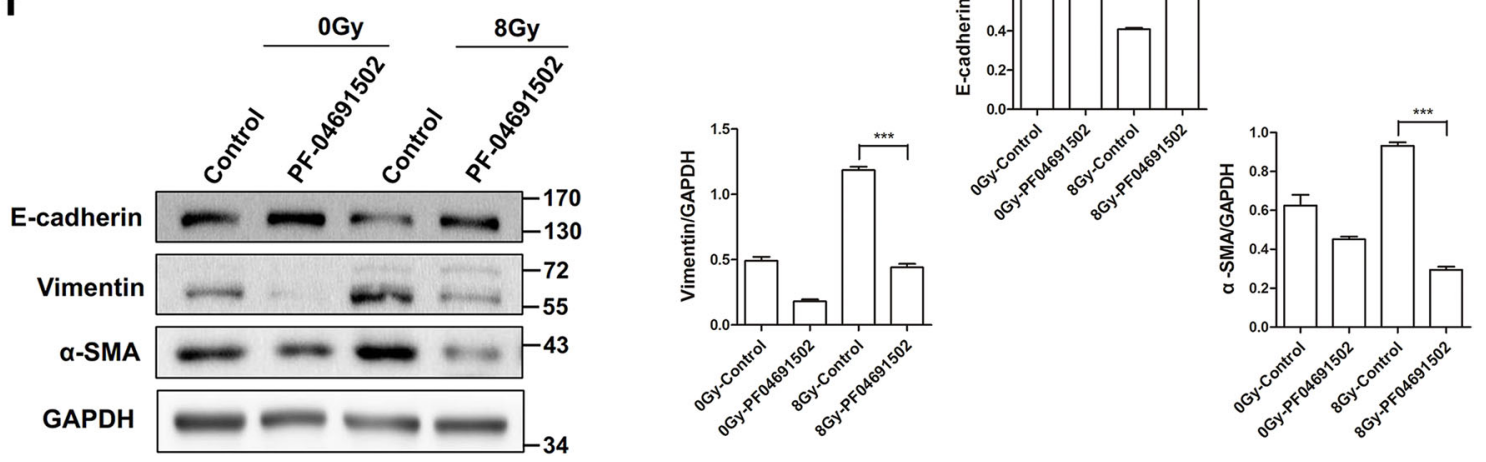

Fig. 3 (See legend on next page.) 
(see figure on previous page)

Fig. 3 Inhibition of AKT attenuates radiation-induced changes in cell morphology and the expression of EMT markers. a, $\mathbf{b}$ Representative western blots and densitometric quantification of phosphorylated AKT on both $\$ 473$ and T308 at different times and with different radiation doses. $\mathbf{c}$ Immunofluorescence staining for p-AKT (green) and DAPI staining (blue) in nonirradiated control and irradiated cells at $1 \mathrm{~h}$ postirradiation. Scale bar represents $50 \mu \mathrm{m}$. $\mathbf{d}-\mathbf{f}$ RLE-6TN cells were incubated with/without the selective AKT inhibitor PF-04691502 for $2 \mathrm{~h}$ before irradiation with $8 \mathrm{~Gy}$. $\mathbf{d}$, $\mathbf{f}$ Western blot analysis of p-AKT, E-cadherin, vimentin, and a-SMA expression at 1 or $48 \mathrm{~h}$ postirradiation. e Phase contrast microscopy images of cells at $48 \mathrm{~h}$ after treatment with $8 \mathrm{~Gy}$ irradiation. Scale bar represents $100 \mu \mathrm{m}$. All the above western blot analyses use GAPDH or AKT as the loading control. The data are presented as the mean $\pm \operatorname{SEM}(n=3) .{ }^{*} P<0.05$, ${ }^{* *} P<0.01$, and ${ }^{* *} P<0.001$ vs. nonirradiated control

\section{Amlexanox protected cells and mice against lethal radiation damage}

To investigate whether Amlexanox exerts protective effects in normal cells and mice, we assessed cell survival in response to different doses of radiation. It was found that Amlexanox significantly increased cell survival after $0,2,4$, and $8 \mathrm{~Gy}$ irradiation (Fig. 9a), which was also confirmed by CCK-8 assays (Fig. 9b). Next, we observed that radiation-induced cell apoptosis in RLE-6TN cells was significantly inhibited by Amlexanox treatment (Fig. 9c). Then, we treated mice with $50 \mathrm{mg} / \mathrm{kg}$ Amlexanox before irradiation and found that animal survival was significantly increased in both the 7.5 and $8.5 \mathrm{~Gy}-$ irradiated groups (Fig. 9d, e).

\section{Discussion}

As a serious complication of thoracic radiotherapy, RIPF is characterized by excessive fibroblast proliferation and massive deposition of extracellular matrix and causes severe physiologic abnormalities and chronic respiratory failure in patients ${ }^{28,29}$. Although fibroblast/myofibroblast activation has been recognized as an important contributor, the exact mechanisms underlying lung fibrosis remain elusive. Recently, experiments using genetic cell fate tracking of lung epithelial cells were performed to investigate this issue in a mouse model of lung fibrosis. These studies found that approximately $30-50 \%$ of murine lung fibroblasts in lung fibrosis were derived from epithelial cells that had undergone EMT as identified by genetic tagging ${ }^{30-32}$. Emerging evidence suggests that injured epithelial cells suffering EMT are important sources of myofibroblasts in fibrosis ${ }^{33}$. In this study, we confirmed that radiation-induced EMT in normal alveolar epithelial cells. Our results showed time-dependent and dose-dependent increases in the expression of TBK1, p$\mathrm{AKT}$, and p-ERK after radiation, and blockage of these proteins reversed radiation-induced EMT, suggesting that they play a crucial role in this process. To the best of our knowledge, this is the first report showing that TBK1 is upstream of ERK and the TBK1-AKT-ERK signaling pathway promotes radiation-induced EMT in normal alveolar epithelial cells.

Recently, cumulative studies have demonstrated that the occurrence of radiation-induced EMT in malignant cancer cells is closely related to their invasive potential. In contrast, only a few groups have conducted studies on normal alveolar epithelial cells undergoing radiationinduced $\mathrm{EMT}^{13,16}$. It is well known that EMT has been classified into three subtypes according to its functional consequences and biomarkers: Type 1 EMT is EMT during implantation, embryogenesis, and organ development; Type 2 EMT is concerned with tissue regeneration and organ fibrosis; Type 3 EMT is associated with cancer progression and metastasis ${ }^{34,35}$. Radiation is a doubleedged sword that not only kills tumor cells but can also damage normal tissues. Here, we sought to study the underlying mechanism of radiation-induced Type 2 EMT in normal alveolar epithelial cells. Nagarajan et al. ${ }^{13}$ first reported radiation-induced EMT in normal rat type II alveolar RLE-6TN cells. Then, Xiong et al. ${ }^{16}$ examined radiation-induced EMT in normal mouse alveolar epithelial MLE 12 cells. In the present study, we demonstrated that radiation-induced EMT is closely related to different radiation doses and time points. In addition to traditional EMT markers, we also quantified the number of cells with obvious morphology changes according to three criteria: swelling, elongation, and extension of pseudopodia from cells vs. a cuboidal appearance. From 0 to $10 \mathrm{~Gy}$, the number of cells undergoing EMT was highest in the $8 \mathrm{~Gy}$ group, while with $10 \mathrm{~Gy}$ irradiation, cell death was induced in more cells. We provided a comprehensive analysis of radiation-induced EMT in normal type II alveolar cells.

TBK1, a noncanonical member of the inhibitor B ( $\mathrm{I} \mathrm{B})$ kinase-related kinase (IKK) family, participates in inflammatory pathways related to activation of $N F-k B$, hosts cytokine production, and plays a crucial role in Rasinduced oncogenesis and in autophagy ${ }^{36-39}$. Additionally, our previous study confirmed that TBK1 promotes radiation-induced Type 3 EMT in lung cancer A549 cells. In the present study, we found that the TBK1 level was increased in a time-dependent and dose-dependent manner after irradiation in normal alveolar RLE-6TN cells. Western blot analysis showed that the loss of TBK1 downregulated the epithelial marker E-cadherin and increased the mesenchymal markers Vimentin and $\alpha$ SMA. Our data suggested that TBK1 induced radiationinduced Type 2 EMT in normal alveolar epithelial cells.

Interestingly, our previous findings indicated that TBK1 promotes radiation-induced EMT through 


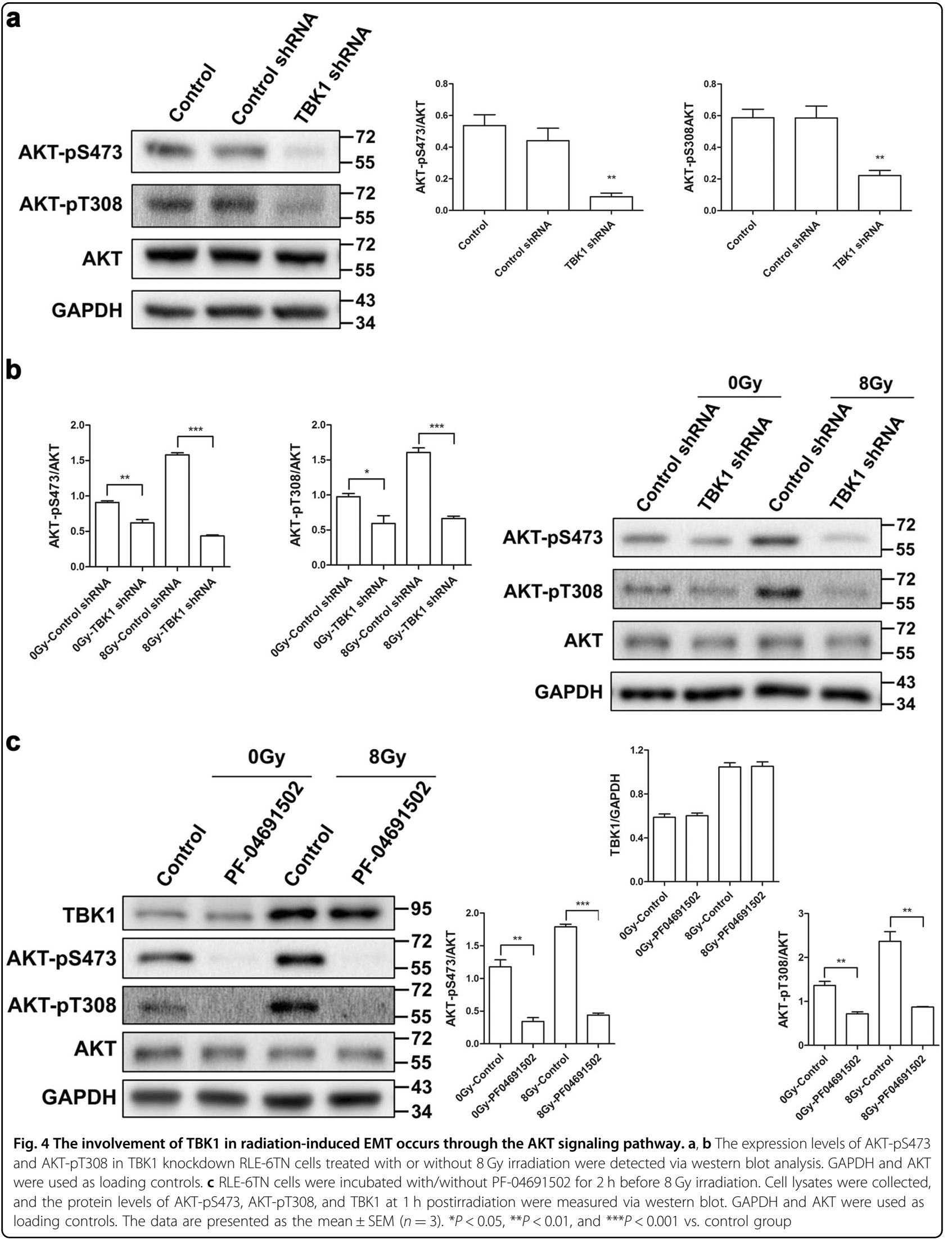




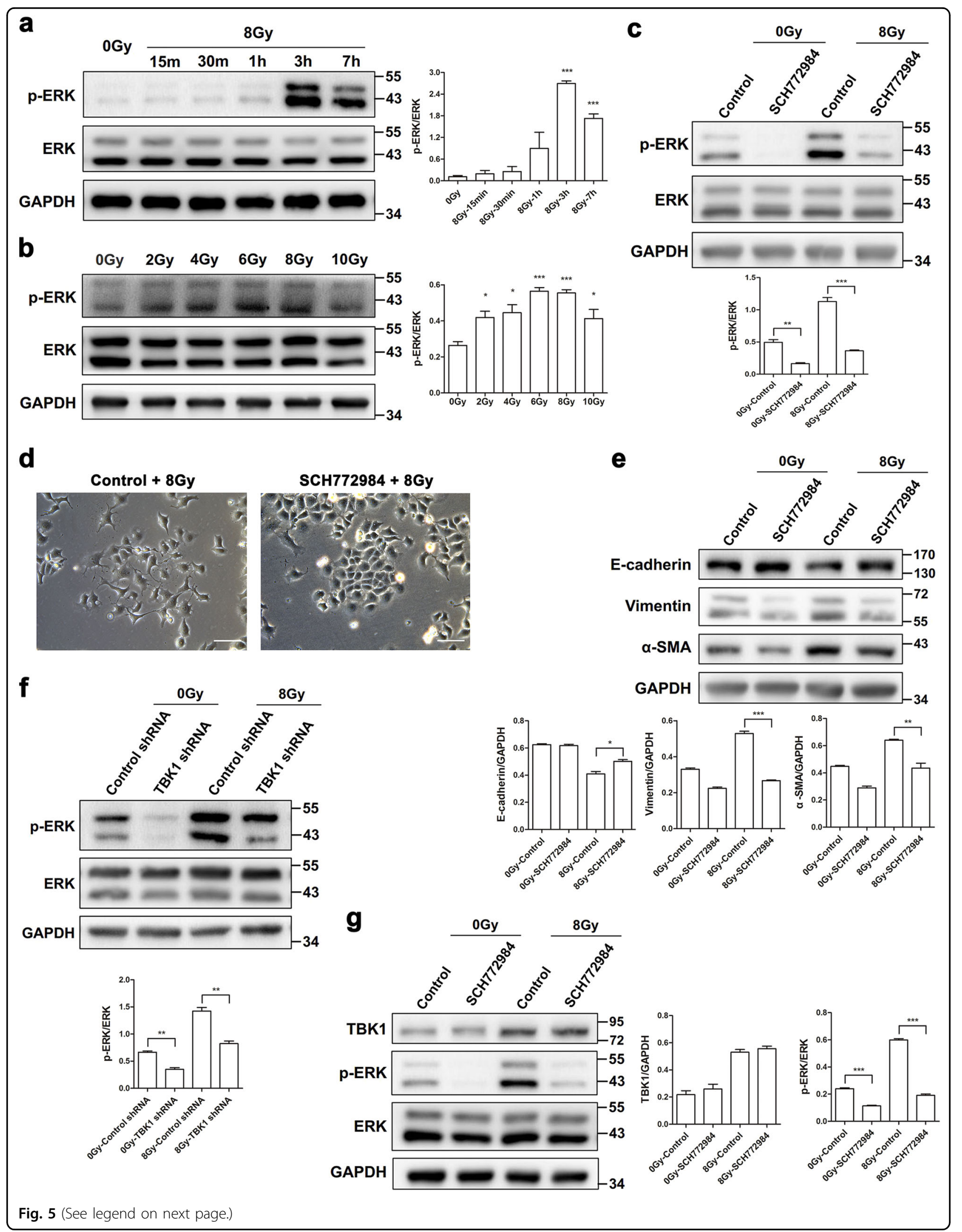


(see figure on previous page)

Fig. 5 The ERK signaling pathway accounts for the regulatory effects of TBK1 in radiation-induced EMT $\mathbf{a}$, $\mathbf{b}$ Representative western blots and densitometric quantification of phosphorylated ERK at different times and with different radiation doses. c-e RLE-6TN cells were incubated with/ without the selective ERK inhibitor SCH772984 for $2 \mathrm{~h}$ before irradiation with 8 Gy. c, e Western blot analysis of p-ERK, E-cadherin, vimentin, and aSMA at 1 or $48 \mathrm{~h}$ postirradiation. $\mathbf{d}$ Phase contrast microscopy images of cells at $48 \mathrm{~h}$ after treatment with $8 \mathrm{~Gy}$ irradiation. Scale bar represents 100 $\mu \mathrm{m}$. $\mathbf{f}$ The expression level of p-ERK in TBK1 knockdown RLE-6TN cells treated with or without 8 Gy irradiation was detected by western blot analysis. g RLE-6TN cells were incubated with/without SCH772984 for $2 \mathrm{~h}$ before irradiation with $8 \mathrm{~Gy}$. Cell lysates were collected, and the protein levels of $\mathrm{p}$ ERK and TBK1 at $3 \mathrm{~h}$ postirradiation were measured by western blot. GAPDH and ERK were used as loading controls. The data are presented as the mean $\pm \operatorname{SEM}(n=3) .{ }^{*} P<0.05,{ }^{*} P<0.01$, and ${ }^{* *} P<0.001$ vs. nonirradiated control
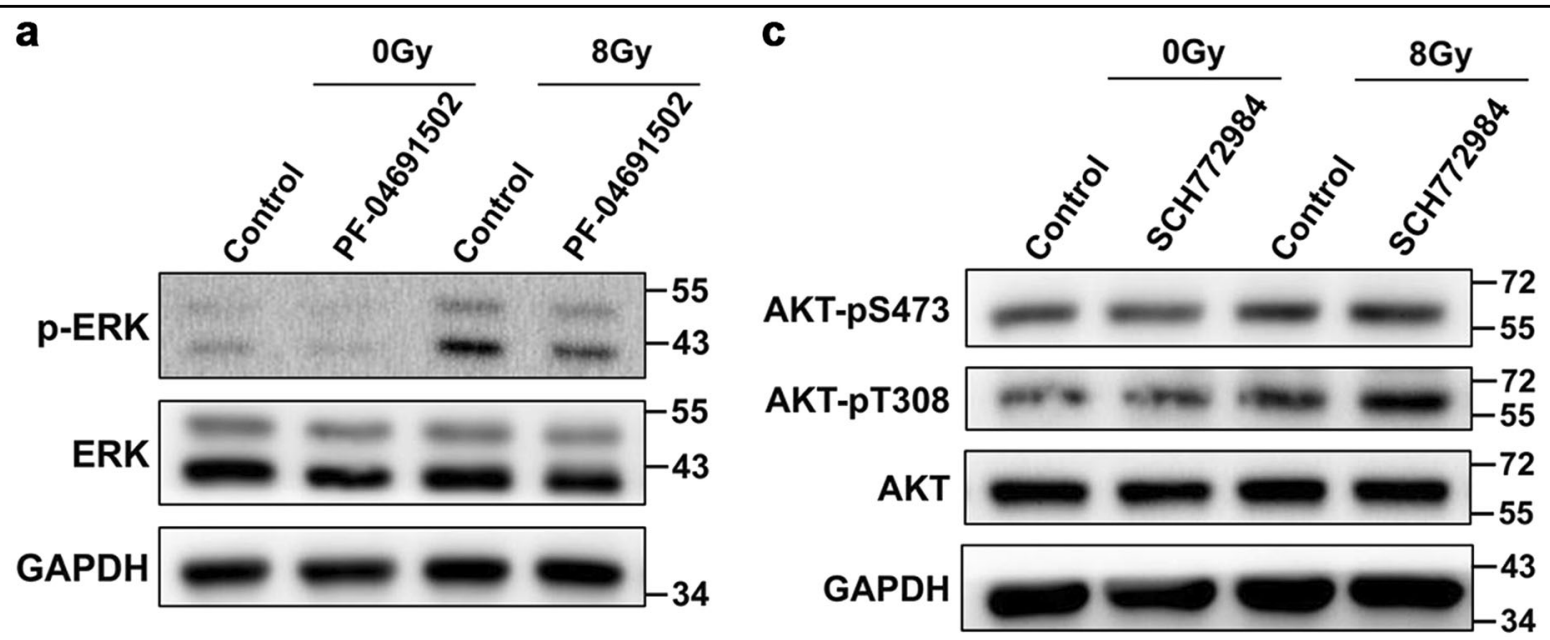

b

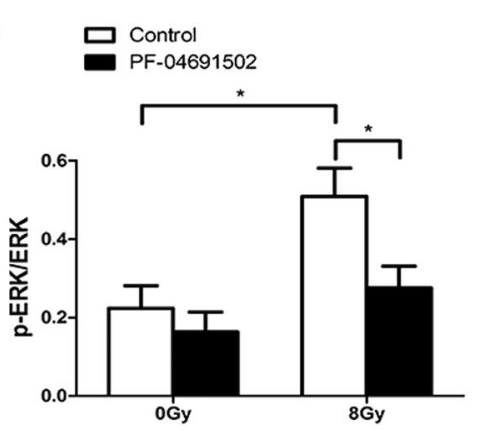

d

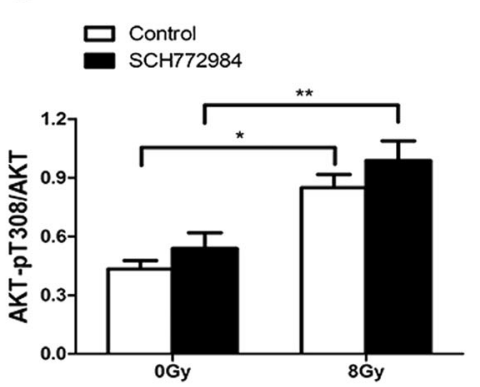

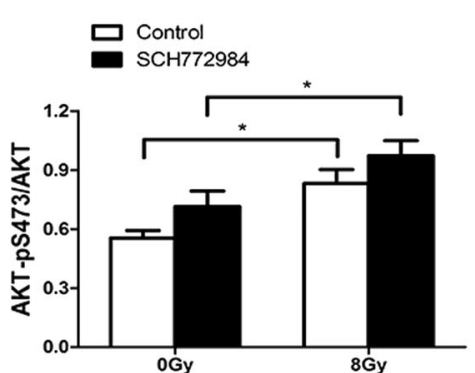

Fig. 6 AKT-mediated ERK signaling is critical for radiation-induced EMT downstream of TBK1. RLE-6TN cells were incubated with/without inhibitor for $2 \mathrm{~h}$ before irradiation with $8 \mathrm{~Gy}$. $\mathbf{a}$, b Representative western blot and densitometric quantification of $\mathrm{p}$-ERK at $3 \mathrm{~h}$ postirradiation. GAPDH and ERK were used as loading controls. c, d Representative western blot and densitometric quantification of AKT-pS473 and AKT-pT308 at $1 \mathrm{~h}$ postirradiation. GAPDH and AKT were used as loading controls. All data are presented as the mean \pm SEM $(n=4)$. One-way ANOVA with Newman-Keuls post hoc analysis was used. ${ }^{*} P<0.05$ and ${ }^{* *} P<0.01$

inactivation of GSK3 $\beta$ and activation of ZEB1 (ref. ${ }^{14}$ ). Previous studies demonstrated that AKT is directly phosphorylated by TBK1 and mediates its prosurvival role $^{26,27}$. The ERK/MAPK and PI3K/Akt pathways mediate Ras mutant-induced Type 3 EMT, which is reversed by either wild-type Ras or MAPK kinase 1 (MEK1) inhibitor ${ }^{40}$. Moreover, activated ERK causes phosphorylation of GSK3 $\beta$, resulting in unbound Snail migrating to the nucleus and eventually leading to EMT $^{13}$. Therefore, we wondered whether TBK1 could regulate radiation-induced Type 2 EMT via AKT and
ERK signaling in normal alveolar epithelial cells. We investigated the response of the AKT and ERK signaling pathway to radiation in RLE-6TN cells. Phosphorylation of both AKT and ERK was triggered in a timedependent and dose-dependent manner in response to irradiation. Moreover, blocking of AKT or ERK abolished radiation-induced EMT, indicating that AKT and ERK signaling are both involved in radiation-induced EMT. The relationship between TBK1 and AKT has been studied for several years, but few report have investigated the crosstalk between TBK1 and ERK. 


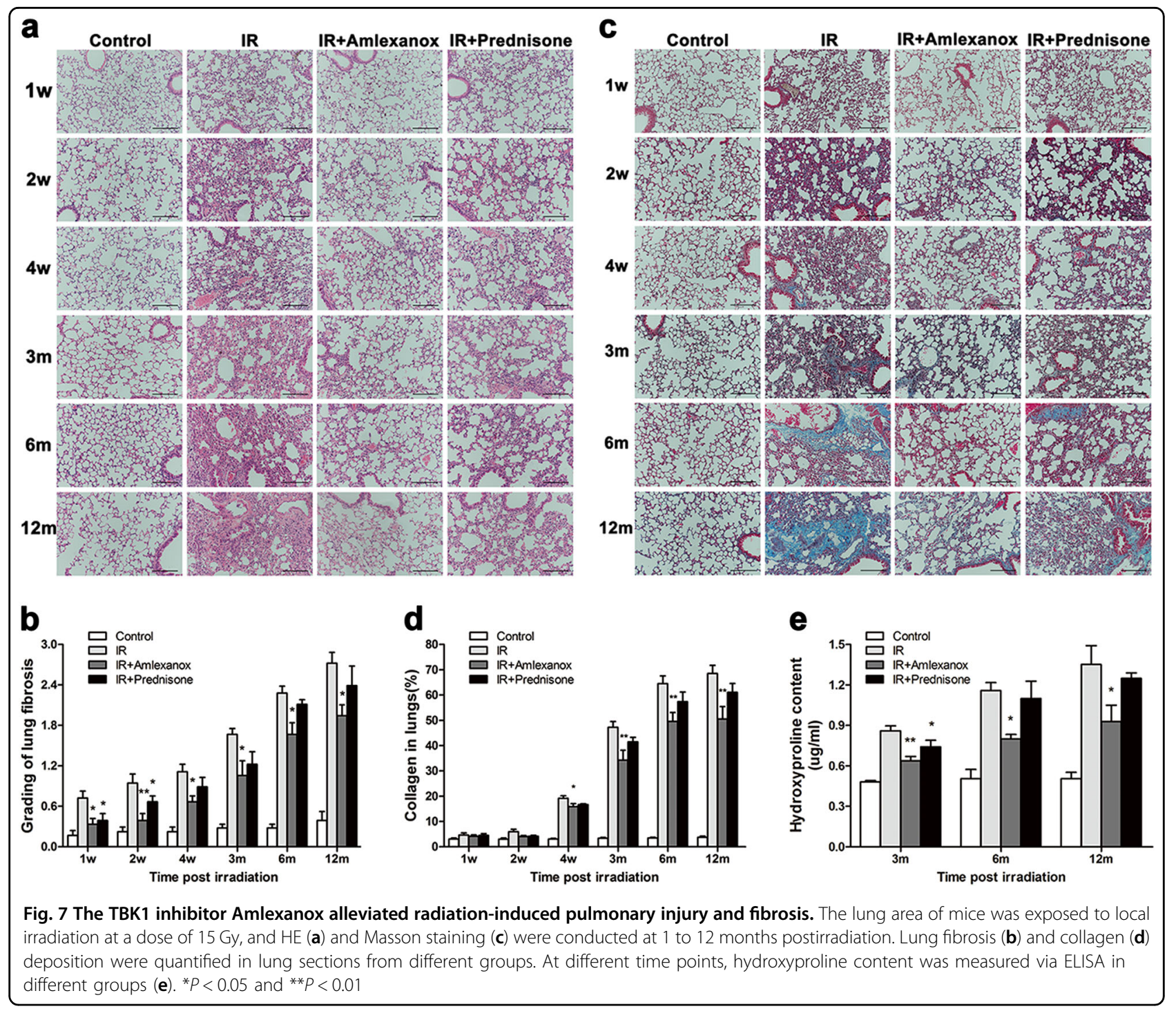

There is no direct evidence showing a role of TBK1 in activation of either AKT or ERK. In TBK1 knockdown cells, we found that radiation-induced phosphorylation of AKT and ERK was inhibited. Conversely, selective inhibitors of AKT or ERK dramatically blocked phosphorylation of AKT and ERK but did not affect the level of TBK1, indicating that AKT and ERK signaling might be downstream of TBK1. As a result, our data suggest that TBK1 signaling may be necessary for regulation of radiation-induced EMT via AKT and ERK signaling in normal alveolar epithelial cells. Then, we focused on the relationship between AKT and ERK. Previously, it was reported that these two signaling pathways exerted opposing effects and that the PI3K-AKT pathway inhibits the Raf-MEK-ERK pathway in muscle cell hypertrophy and breast cancer cell proliferation ${ }^{41,42}$. In contrast, we observed that inhibition of AKT signaling decreased the radiation-induced increase in p-ERK, while blockage of ERK signaling did not obviously change the expression of p-AKT. These data suggest that AKT might exert an upstream regulator of ERK in radiation-induced EMT, but the exact mechanism still needs to be clarified.

According to previous studies and our present findings, radiation-induced EMT in RLE-6TN cells is mediated, in part, through the TBK1-ERK-AKT signaling pathway. However, our study has some limitations. As a crucial signaling factor in EMT, transforming growth factor- $\beta$ (TGF$\beta$ ) is heavily implicated in radiation-induced fibrosis ${ }^{43}$. However, our previous findings suggested a lack of crosstalk between TBK1 signaling and the TGF- $\beta /$ Smad pathway in radiation-induced EMT in lung cancer A549 cells, and a previous study has shown that ERK is less likely to be involved in TGF- $\beta$-induced human alveolar $\mathrm{EMT}^{44}$. In addition, whether ZEB1, Snail, and other major transcription factors, such as TWIST1, participate in this signaling 


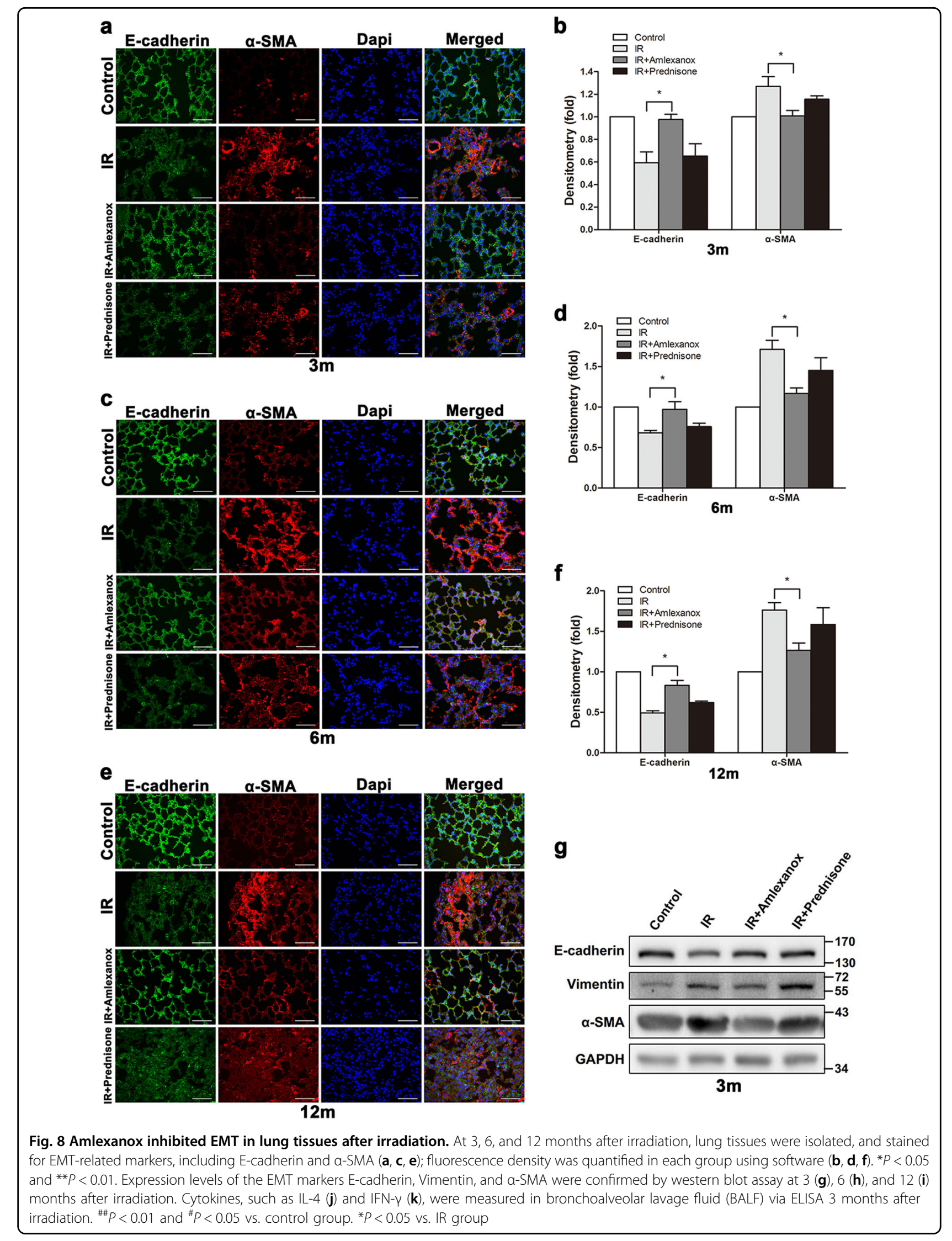



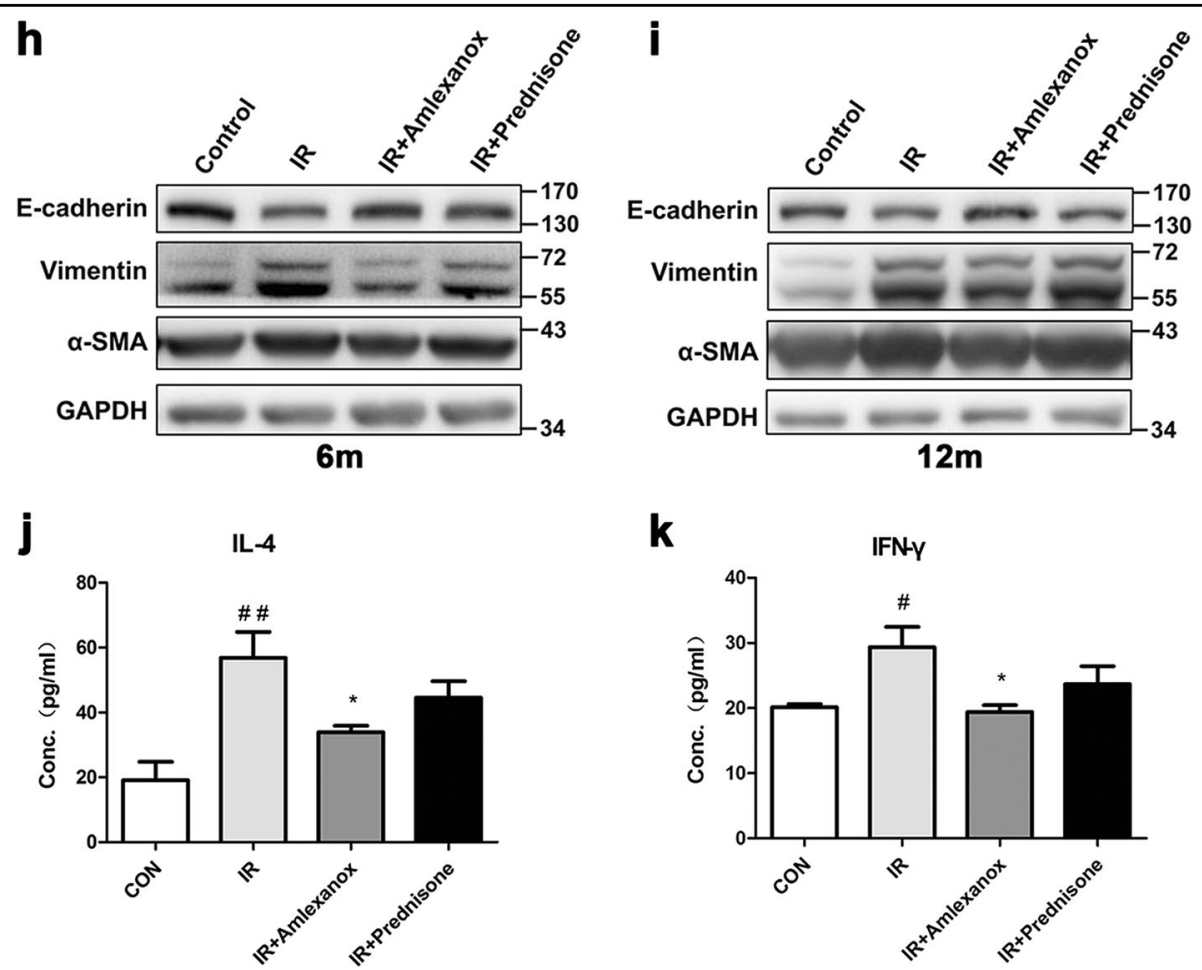

Fig. 8 (See legend on previous page.)
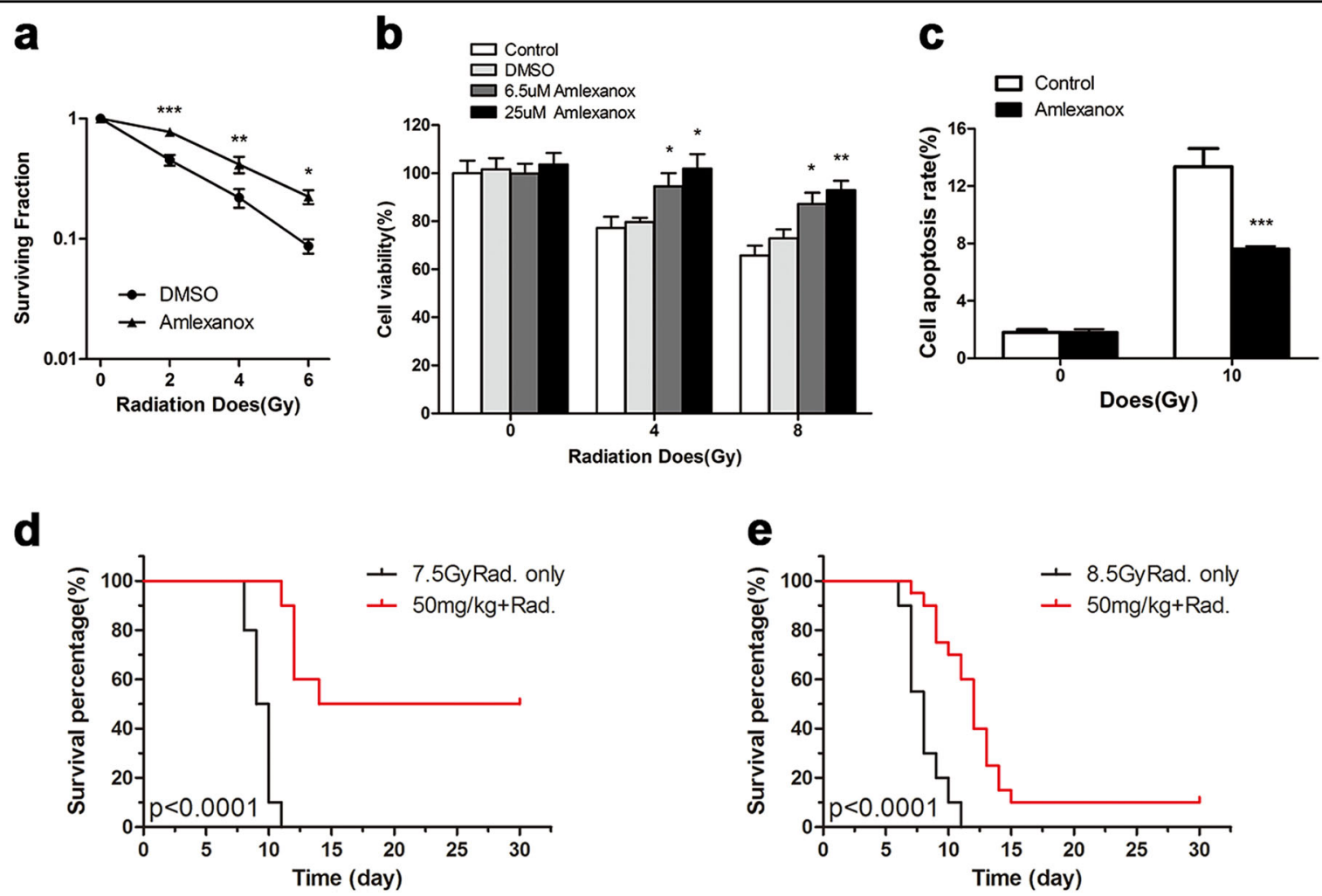

Fig. 9 The TBK1 inhibitor Amlexanox protected cells and mice against lethal radiation damage. After 2, 4, 6, or 8 Gy single-dose irradiation, cell survival was determined in the radiation- and Amlexanox-treated groups via colony formation (a) and CCK-8 (b) assays. Cell apoptosis was measured by flow cytometry $24 \mathrm{~h}$ after $10 \mathrm{~Gy}$ irradiation (c). For in vivo study, BALB/c mice with/without Amlexanox treatment were exposed to 7 or $8.5 \mathrm{~Gy}$ irradiation, and animal survival was monitored for up to 30 days after irradiation (d, e). ${ }^{*} P<0.05,{ }^{* *} P<0.01$, and ${ }^{* * *} P<0.001$ vs. nonirradiated control 
pathway still needs to be validated. Cell culture models are beneficial for exploring the mechanism of EMT, lung fibrosis, and the associated treatment strategies.

The critical role of TBK1 in radiation-induced EMT provided novel therapeutic potential for radiation pulmonary fibrosis. In the present study, by using a RIPI model established by our group, we investigated the possibility of blocking TBK1 to prevent RIPI. Our data confirmed that a TBK1 inhibitor alleviated lung injury as well as fibrosis and inhibited the EMT process. EMT is a major process in fibrosis in many organs, and we also found that TBK1 inhibited EMT and reduced collagen deposition in lung tissues. As Th1 and Th2 balance play critical roles in lung fibrosis, we further examined the impact of TBK1 depletion on the Th1/Th2 cytokine balance in BALF after irradiation ${ }^{45}$. As is shown in our results, the levels of IFN- $\gamma$ and IL-4 in BALF increased after radiation, while TBK1 inhibitor restricted the upregulation of these cytokines. Finally, we found that Amlexanox protects normal cells and mice against irradiation. These data provide novel insights into potential therapy for RIPF in normal tissues, which will be beneficial for delivery of radiotherapy in cancer patients.

In conclusion, we have shown that inhibition of TBK1, AKT, and ERK signaling suppressed radiation-induced EMT in RLE-6TN cells. In particular, TBK1, as a significant upstream signaling molecule, regulates radiationinduced EMT through AKT-ERK signaling. Our in vivo data showed that a TBK1 inhibitor alleviates RIPI and protects mice against IR, providing a novel strategy for preventing RIPI during cancer radiotherap.

\section{Acknowledgements}

This study was supported in part by grants from National Natural Science Foundation of China (No. 11635014, No. 31670861, No. 31700739, and No. 11605289)

\section{Conflict of interest}

The authors declare that they have no conflict of interest.

\section{Publisher's note}

Springer Nature remains neutral with regard to jurisdictional claims in published maps and institutional affiliations.

Received: 16 July 2018 Revised: 25 November 2018 Accepted: 11 December 2018.

Published online: 15 April 2019

\section{References}

1. Chen, W. et al. Cancer statistics in China, 2015. CA Cancer J. Clin. 66, 115-132 (2016).

2. Siegel, R. L., Miller, K. D. \& Jemal, A. Cancer statistics, 2015. CA Cancer J. Clin. 65, 5-29 (2015).

3. Mehta, V. Radiation pneumonitis and pulmonary fibrosis in non-small-cell lung cancer: pulmonary function, prediction, and prevention. Int. J. Radiat. Oncol. Biol. Phys. 63, 5-24 (2005).
4. Ding, N. H., Li, J. J. \& Sun, L. Q. Molecular mechanisms and treatment of radiation-induced lung fibrosis. Curr. Drug Targets. 14, 1347-1356 (2013).

5. Graves, P. R., Siddiqui, F., Anscher, M. S. \& Movsas, B. Radiation pulmonary toxicity: from mechanisms to management. Semin. Radiat. Oncol. 20, 201-207 (2010).

6. Benveniste, M. F. et al. New era of radiotherapy: an update in radiationinduced lung disease. Clin. Radiol. 68, e275-e290 (2013).

7. Koukourakis, M. I. Radiation damage and radioprotectants: new concepts in the era of molecular medicine. Br. J. Radiol. 85, 313-330 (2012).

8. Bentzen, S. M. Preventing or reducing late side effects of radiation therapy: radiobiology meets molecular pathology. Nat. Rev. Cancer 6, 702-713 (2006).

9. Marmai, C. et al. Alveolar epithelial cells express mesenchymal proteins in patients with idiopathic pulmonary fibrosis. Am. J. Physiol. Lung C 301, L71-L78 (2011).

10. Balli, D. et al. Foxm1 transcription factor is required for lung fibrosis and epithelial-to-mesenchymal transition. EMBO J. 32, 231-244 (2013).

11. Sime, P. J. The antifibrogenic potential of PPAR gamma ligands in pulmonary fibrosis. J. Invest. Med. 56, 534-538 (2008).

12. Radisky, D. C. Epithelial-mesenchymal transition. J. Cell Sci. 118, 4325-4326 (2005).

13. Nagarajan, D., Melo, T., Deng, Z., Almeida, C. \& Zhao, W. ERK/GSK3beta/Snail signaling mediates radiation-induced alveolar epithelial-to-mesenchymal transition. Free Radic. Biol. Med. 52, 983-992 (2012).

14. Liu, W. et al. Inhibition of TBK1 attenuates radiation-induced epithelialmesenchymal transition of A549 human lung cancer cells via activation of GSK-3beta and repression of ZEB1. Lab. Invest. 94, 362-370 (2014).

15. Almeida, C. et al. The role of alveolar epithelium in radiation-induced lung injury. PLoS ONE 8, e53628 (2013).

16. Xiong, $\mathrm{S}$. et al. Regulatory $\mathrm{T}$ cells promote beta-catenin-mediated epitheliumto-mesenchyme transition during radiation-induced pulmonary fibrosis. Int. J. Radiat. Oncol. Biol. Phys. 93, 425-435 (2015).

17. Kalluri, R. \& Neilson, E. G. Epithelial-mesenchymal transition and its implications for fibrosis. J. Clin. Invest. 112, 1776-1784 (2003).

18. Lamouille, S., Xu, J. \& Derynck, R. Molecular mechanisms of epithelialmesenchymal transition. Nat. Rev. Mol. Cell Biol. 15, 178-196 (2014).

19. Peinado, H., Olmeda, D. \& Cano, A. Snail, Zeb and bHLH factors in tumour progression: an alliance against the epithelial phenotype? Nat. Rev. Cancer 7, 415-428 (2007).

20. Lin, J. et al. miR-200c enhances radiosensitivity of human breast cancer cells. J. Cell. Biochem. 114, 606-615 (2013).

21. Hurteau, G. J., Carlson, J. A., Spivack, S. D. \& Brock, G. J. Overexpression of the microRNA hsa-miR-200c leads to reduced expression of transcription factor 8 and increased expression of E-cadherin. Cancer Res. 67, 7972-7976 (2007).

22. Coco, S. et al. Afatinib resistance in non-small cell lung cancer involves the PI3KJAKT and MAPKJERK signalling pathways and epithelial-to-mesenchymal transition. Target Oncol. 10, 393-404 (2015).

23. Zhang, Y. Q. et al. Over-expressed twist associates with markers of epithelial mesenchymal transition and predicts poor prognosis in breast cancers via ERK and Akt activation. PLOS ONE 10, e0135851 (2015).

24. Yu, H., Zhang, L. \& Liu, P. CXCR7 signaling induced epithelial-mesenchymal transition by AKT and ERK pathways in epithelial ovarian carcinomas. Tumour Biol. 36, 1679-1683 (2015).

25. Wang, Z. et al. STYK1 promotes epithelial-mesenchymal transition and tumor metastasis in human hepatocellular carcinoma through MEK/ERK and PI3K AKT signaling. Sci. Rep. 6, 33205 (2016).

26. Xie, X. D. et al. I kappa B kinase epsilon and TANK-binding kinase 1 activate AKT by direct phosphorylation. Proc. Natl. Acad. Sci. USA. 108, 6474-6479 (2011).

27. Ou, Y. H. et al. TBK1 directly engages Akt/PKB survival signaling to support oncogenic transformation. Mol. Cell 41, 458-470 (2011).

28. Marks, L. B. The pulmonary effects of thoracic irradiation. Oncology (Williston Park) 8, 89-106 (1994). discussion 100, 103-104.

29. Marks, L. B. et al. Radiation-induced lung injury. Semin. Radiat. Oncol. 13, 333-345 (2003).

30. Kim, K. K. et al. Alveolar epithelial cell mesenchymal transition develops in vivo during pulmonary fibrosis and is regulated by the extracellular matrix. Proc. Natl. Acad. Sci. USA 103, 13180-13185 (2006).

31. Tanjore, $H$. et al. Contribution of epithelial-derived fibroblasts to bleomycininduced lung fibrosis. Am. J. Respir. Crit. Care Med. 180, 657-665 (2009). 
32. Degryse, A. L. et al. Repetitive intratracheal bleomycin models several features of idiopathic pulmonary fibrosis. Am. J. Physiol. Lung C 299, L442-L452 (2010).

33. Selman, M. \& Pardo, A. Role of epithelial cells in idiopathic pulmonary fibrosis: from innocent targets to serial killers. Proc. Am. Thorac. Soc. 3, 364-372 (2006).

34. Huang, R. Y., Guilford, P. \& Thiery, J. P. Early events in cell adhesion and polarity during epithelial-mesenchymal transition. J. Cell Sci. 125, 4417-4422 (2012).

35. Kalluri, R. \& Weinberg, R. A. The basics of epithelial-mesenchymal transition. J. Clin. Invest. 119, 1420-1428 (2009).

36. Shen, R. R. \& Hahn, W. C. Emerging roles for the non-canonical IKKs in cancer. Oncogene 30, 631-641 (2011).

37. Chau, T. L. et al. Are the IKKs and IKK-related kinases TBK1 and IKK-epsilon similarly activated? Trends. Biochem. Sci. 33, 171-180 (2008).

38. Kim, J. Y. et al. Dissection of TBK1 signaling via phosphoproteomics in lung cancer cells. Proc. Natl. Acad. Sci. USA 110, 12414-12419 (2013).
39. Weidberg, H. \& Elazar, Z. TBK1 mediates crosstalk between the innate immune response and autophagy. Sci. Signal. 4, pe39 (2011).

40. Janda, E. et al. Ras and TGF[beta] cooperatively regulate epithelial cell plasticity and metastasis: dissection of Ras signaling pathways. J. Cell Biol. 156, 299-313 (2002).

41. Rommel, C. et al. Differentiation stage-specific inhibition of the Raf-MEK-ERK pathway by Akt. Science 286, 1738-1741 (1999).

42. Zimmermann, S. \& Moelling, K. Phosphorylation and regulation of Raf by Akt (protein kinase B). Science 286, 1741-1744 (1999).

43. Straub, J. M. et al. Radiation-induced fibrosis: mechanisms and implications for therapy. J. Cancer Res. Clin. 141, 1985-1994 (2015).

44. Kasai, H., Allen, J. T., Mason, R. M., Kamimura, T. \& Zhang, Z. TGF-beta1 induces human alveolar epithelial to mesenchymal cell transition (EMT). Respir. Res. $\mathbf{6}$, 56 (2005).

45. Wynn TA. Fibrotic disease and the $\mathrm{TH} 1 / \mathrm{TH} 2$ paradigm. Nat. Rev. Immunol. 4 , 583-594 (2004). 